\title{
The Influence of Desquamative Gingivitis on Periodontal Health
}

\author{
Monica Monea1, Tudor Hănțoiu¹, Alexandra Stoica1', Ramona Vlad², Alexandru Sitaru¹ \\ 1 Department of Odontology and Oral Pathology, Faculty of Dental Medicine, University of Medicine and Pharmacy, Tîrgu Mureș, Romania \\ 2 Master student, University of Medicine and Pharmacy, Tîrgu Mureș, Romania
}

\section{CORRESPONDENCE}

\section{Monica Monea}

Str. Gheorghe Marinescu nr. 38

540039 Tîrgu Mureș, Romania

Tel: +40 745177369

E-mail: monea_monica@yahoo.com

\section{ARTICLE HISTORY}

Received: 3 March, 2017

Accepted: 9 March, 2017
Tudor Hănțoiu • Str Gheorghe Marinescu nr 38 540139 Tîrgu Mureș, Romania. Tel: +40 265215551 Alexandra Stoica • Str. Gheorghe Marinescu nr. 38 , 540139 Tîrgu Mureș, Romania. Tel: +40 265215551 Ramona Vlad • Str. Gheorghe Marinescu nr. 38 540139 Tîrgu Mureș, Romania. Tel: +40 265215551 Alexandru Sitaru • Str. Gheorghe Marinescu nr. 38 540139 Tîrgu Mures, Romania. Tel: +40 265215551

\begin{abstract}
Background: Desquamative gingivitis (DG) is a non-plaque-induced, blistering and painful condition occurring most frequently on the labial aspect of the attached gingiva of anterior teeth. The incidence of DG is highest around 50 years of age, and usually indicates the presence of oral or systemic diseases. The purpose of our study was to determine the impact of DG on periodontal health by recording the plaque index, gingival index and gingival bleeding index in a group of patients with DG, compared to healthy controls. Materials and methods: Recordings of specific indices were performed in a group of 26 patients with DG and compared with 24 healthy individuals. These were followed by radiographic examinations in order to assess the loss of marginal alveolar bone. Results: The results showed that patients with DG had a statistically significant increase in periodontal indices, with more gingival inflammation and plaque retention compared to the control group $(p<0.05)$. The highest scores for gingival inflammation were recorded in patients with DG, but on radiographic evaluation the difference was related only to gender, men being more affected by alveolar bone loss in both groups ( $p$ $<0.05$ ). Conclusions: The incidence and severity of gingival inflammation proved to be higher in patients with DG, which calls for better preventive and maintenance treatment protocols in this group of patients. Early diagnosis and initial-phase periodontal treatment are very important in preventing further tissue breakdown.
\end{abstract}

Keywords: desquamative gingivitis, oral lichen planus, periodontal disease, dental plaque

\section{INTRODUCTION}

Desquamative gingivitis (DG) is a non-plaque-induced, blistering and painful condition occurring most frequently on the labial aspect of the attached gingiva of anterior teeth. This is not a specific disease, but rather a gingival response associated with a variety of systemic conditions. ${ }^{1,2}$ The incidence of DG is at its peak around 50 years of age, and usually indicates the presence of oral or systemic diseases, such as lichen planus, leukoplakia, lupus erythematosus, psoriasis, allergic and neoplastic lesions. Therefore, the dental practitioner has to complete an important role in the identification of the condition that lies behind each case of DG, based on a thorough differential diagnosis. The pathogenesis of DG-associated diseases can be cell-mediated (as OLP, lupus erythematosus) or 
auto-antibody-mediated (as pemphigus vulgaris, mucous membrane pemphigoid). The first usually induce alterations in the epithelial thickness or hyperkeratosis, while the latter lead to blister formation. Oral lichen planus (OLP) is a chronic mucocutaneous disease that can affect different sites of the oral mucosa, including the gingiva, at this level determining a series of pathological alterations, including DG. It is considered to be responsible for $24-45 \%$ of cases of DG, but may reach up to $75 \% .3,4$

OLP is determined by auto-cytotoxic T-lymphocytes that induce apoptosis of the epithelial cells via tumor necrosis factor-alpha, causing the formation of white plaques or striations, associated with blisters or bullae of the gingiva. ${ }^{5}$ It has a $3 / 2$ predilection for adult women, with an oral incidence of $0.1-2.2 \% .{ }^{3}$ Pemphigus vulgaris is an autoantibody-mediated disease usually accompanied by acantholysis, the involvement of oral gingiva being noted in the early stages in up to $70 \%$ of patients. ${ }^{6}$

The purpose of our study was to determine the impact of DG on periodontal health, by measuring specific indicators such as plaque accumulation, gingival inflammation, gingival bleeding and periodontal pocket depth, in a group of patients diagnosed with oral lesions or systemic diseases in comparison to healthy controls. The null hypothesis to be tested is that DG has no influence on periodontal health.

\section{MATERIALS AND METHODS}

This study was carried out in accordance with the Helsinki Declaration and the standards of the Ethical Committee for Scientific Research of our university. A total of 26 patients with oral lesions or systemic diseases associated with DG, enrolled in the database of the Department of Odontology and Oral Pathology of our faculty, had been selected and signed an informed consent to take part in this investigation. For comparison, a healthy age- and sex-matched control group of 24 patients was selected. Patients over 18 years of age, non-smokers, without antibiotic therapy for the last 2 months, with at least 16 teeth available for dental scoring and a history of oral lesions or systemic disease associated with DG were included. As exclusion criteria, tooth destruction due to dental caries, extensive prosthetic restorative work and periodontal disease were used.
All the recordings were made twice, at an interval of one week, in order to have an objective result.

We used the following indices: the Quigley-Hein Plaque Index (PI), the Modified Gingival Index (MGI) and the Sulcus Bleeding Index (SBI), according to their scoring systems. For the PI we used the following criteria: 0 - no visible dental plaque, 1 - isolated spots of plaque, mostly on cervical areas, 2 - a continuous $1 \mathrm{~mm}$ wide layer of plaque at cervical level, 3 - a continuous layer of plaque covering less than $1 / 3$ of the dental surface, $4-$ a continuous layer of plaque covering $1 / 3-2 / 3$ of the dental surface, 5 - more than $2 / 3$ of the dental surface are covered by plaque. The scores for MGI: 0 - no inflammation, 1 - localized, slight inflammation, 2 - inflammation extended to the interdental papilla or gingival margin, 3 - moderate inflammation, edema, hypertrophy of the marginal gingiva, 4 - severe inflammation, ulcerative lesions, bleeding of the gingival margin. The scores for SBI: 0 - normal gingiva, absence of edema or bleeding, 1 - normal gingiva with bleeding on probing, 2 - no edema, color alteration and bleeding on probing, 3 edema, color alteration and bleeding on probing, 4 - color and volume alteration associated with bleeding on probing, 5 - intense alteration of gingival aspect and bleeding. For each case a panoramic radiographic investigation was indicated, in order to evaluate the loss of marginal alveolar bone.

All recorded data were analyzed using the statistical software SPSS version 16 (SPSS Inc, Chicago, USA); the comparison between the study and control group was determined with ANOVA variation test and Student's t test. The level of significance was set at a value of $\mathrm{p}<0.05$.

\section{RESULTS}

The ANOVA analysis applied to demographic data collected from the groups showed the absence of statistically significant differences regarding the age and sex of patients $(p>0.05)$. In the study group there were 15 women and 9 men, with an age interval between $31-56$ years (43 \pm 12.7 ), compared to the control group, with 14 women and 10 men and an age interval between $24-41$ years (31 $\pm 8.65)$. In the study group, 8 cases of DG were associated with the presence of oral leukoplakia, 5 cases with OLP, 8 cases with psoriasis and 5 cases with lupus erythematous (Table1).

TABLE 1. Demographic and clinical characteristics of the study group

\begin{tabular}{lcccc}
\hline Age and sex & DG /leukoplakia & DG / OLP & DG / psoriasis & DG / LE \\
\hline 31-56 years, 15 women/11 men & 8 cases (30.7\%) & 5 cases (19.23\%) & 9 cases (34.6\%) & 4 cases (15.38\%) \\
\hline
\end{tabular}


TABLE 2. Recordings of the clinical parameters

\begin{tabular}{lccc}
\hline Clinical parameter & Study group & Control group & Clinical examination \\
\hline $\mathrm{MGI}$ & $1.78 \pm 0.32$ & $0.63 \pm 0.12^{*}$ & $1^{\text {st }}$ \\
& $1.79 \pm 0.33$ & $0.69 \pm 0.21^{*}$ & $2^{\text {nd }}$ \\
$\mathrm{SBI}$ & $0.71 \pm 0.16$ & $0.59 \pm 0.11$ & $1^{\text {st }}$ \\
& $0.79 \pm 0.24$ & $0.63 \pm 0.16$ & $2^{\text {nd }}$ \\
$\mathrm{PI}$ & $2.48 \pm 0.40$ & $1.65 \pm 0.31^{*}$ & $1^{\text {st }}$ \\
& $2.69+/-0.48$ & $1.86 \pm 0.46^{*}$ & $2^{\text {nd }}$ \\
\hline
\end{tabular}

Statistical analysis using Student's t-test applied on the results of MGI measurements show significant differences between the study and control group $(\mathrm{p}<0.05)$. The same observation was recorded for PI, but in the case of SBI there were no statistically significant differences between the groups ( $p>0.05$ ) (Table 2).

The highest score for PI was recorded in cases with ulcerative DG, while for erythematous and mixed clinical aspect the scores were lower. The radiographic evaluation was based on panoramic radiographs examined under magnification, in order to measure the distance from the cement-enamel junction to the alveolar bone crest ( Table 3). The comparison between genders in both groups showed statistically significant differences $(\mathrm{p}<0.05)$, with more bone loss in men.

\section{DISCUSSION}

The presence of oral lesions associated with a painful oral hygiene protocol might have a negative impact on periodontal health, increasing the possibility of dental plaque accumulation and the development of gingival inflammation. Some studies reported significantly worse periodontal indices in patients with DG compared to healthy controls. ${ }^{7-12}$ Akman et al. found that the periodontal status was worse in patients with pemphigus vulgaris; this was confirmed by LoperzJornet and Camacho-Alonso after a similar investigation in a group of patients with DG and OLP. ${ }^{7,8}$ There are also studies that report slight differences between patients exhibit- ing DG in comparison to healthy individuals. Nevertheless, the severity of gingival inflammation is positively correlated with the extent and clinical aspect of DG. ${ }^{13,14}$

In our study, the ulcerative form of DG was associated with the highest scores for PI and MGI, but there were no statistically significant differences in the SBI scores. The amount of horizontal and vertical bone loss was higher in men compared to women in both groups, which could be related to the presence of associated risk factors for gingival inflammation such as smoking and alcohol intake.

The presence of pain during oral hygiene procedures could be another factor with negative impact on the quality of life in patients with DG, an important aspect that needs to be investigated. In this respect, the association of pain and DG correlated to periodontal indices and the longterm monitoring of patients under pain-control medication could offer important information regarding the influence of DG on periodontal health. The influence of OLP on the periodontal supporting tissues was investigated by Ertugrul et al., who used the levels of matrix metalloproteinase released by gingival fibroblasts. ${ }^{15}$ These are zinc-containing enzymes responsible for collagen degradation, which is the main component of the extracellular matrix of periodontal tissues. MMP-1 is a key regulator of remodeling processes in the gingiva, and MMP-9 is important in the degradation of the basal membrane. The evaluation of gingival fluid and saliva samples from patients with OLP and DG showed higher values of both MMPs, which suggested that OLP and DG might affect periodontal health. Therefore, an early

TABLE 3. Gender distribution of bone loss in the study and control group

\begin{tabular}{lccc}
\hline Group & Gender & Horizontal bone loss & Vertical bone loss \\
\hline Study group & Men & $28.5 \%$ & $14.2 \%^{*}$ \\
& Women & $11 \%$ & $11 \%$ \\
Control group & Men & $21.2 \%$ & $11.3 \%$ \\
& Women & $18 \%$ & $9 \%$ \\
\hline
\end{tabular}


diagnosis and treatment is an important preventing attitude for further tissue breakdown in these cases.

The patients from both the study and control group enrolled in our study showed an unsatisfactory level of oral hygiene, which is an alarming sign regarding dental health education in the general population. The accumulation of dental plaque acts as an irritating factor that might favor the development of oral lesions and stimulate the autoimmune response.

As DG is a non-plaque-induced form of gingival inflammation, dental practitioners should consider it an early oral manifestation of a systemic disease. If this is accompanied by a poor oral hygiene, then all conditions are present for further development of periodontal disease. Therefore, patients exhibiting DG should be informed about this potential risk and the benefits of a long-term maintenance oral hygiene protocol should be emphasized, in order to stop disease progression.

\section{CONCLUSIONS}

The incidence and severity of periodontal disease proved to be higher in patients with chronic gingival conditions, which calls for better preventive and maintenance treatment protocols in this group of patients. Early diagnosis and treatment, such as the removal of local irritating factors like calculus and dental plaque, are very important in preventing periodontal breakdown. Further studies are required in order to determine whether the inflammatory alterations in the periodontal tissues are related to the oral disease associated to DG or only to the accumulation of dental plaque alone.

\section{ACKNOWLEDGEMENT}

This study was conducted under the framework of the research grant no. 3688/01.04.2016, financed by SC COSA-
MEXT SRL Tîrgu Mureș and developed by the University of Medicine and Pharmacy of Tîrgu Mureș.

\section{CONFLICT OF INTEREST}

Nothing to declare.

\section{REFERENCES}

1. Lo Russo L, Fedele S, Guiglia R, et al. Diagnostic pathways and clinical significance of desquamative gingivitis. J Periodontol. 2008;79:4-24.

2. Leao J, Ingafon M, Khan A, Scully C, Porter S. Desquamative gingivitis: retrospective analysis of disease associations of a large cohort. Oral Diseases. 2008;14:556-560.

3. Lo Russo L, Fierro G, Guiglia R, et al. Epidemiology of desquamative gingivitis: evaluation of 125 patients and review of the literature. Int $J$ Dermatol. 2009;48:1049-1052

4. Roopashree MR, Goudhalekar RV, Shashikanth MC, George J, Thippeswamy SH, Skula A. Pathogenesis of oral lichen planus: a review. J Oral Pathol Med. 2010;39:729-734

5. Said S, Golitz L. Vesiculobullous eruptions of the oral cavity. Otolaryngol Clin North Am. 2011;44:133-160.

6. Sirois D, Leigh JE, Sollecito TP. Oral pemphigus vulgaris preceding cutaneous lesions: recognition and diagnosis. J Am Dent Assoc. 2000;131:1156-1160.

7. Akman A, Kacaroglu H, Yilmaz E, Alpsoy E. Periodontal status in patients with pemphigus vulgaris. Oral Dis. 2008;14:640-643.

8. Lopez-Jornet P, Camaco-Alonso F. Periodontal conditions in patients with oral lichen planus: a pilot study. Quintessence Int. 2012;43:147-152.

9. Lo Russo L, Gallo C, Pellegrino G, et al. Periodontal clinical and microbiological data in desquamative gingivitis patients. Clin Oral Investig. 2014;18:917-925.

10. Scully C, Carrozzo M. Oral mucosal disease: lichen planus. Br J Oral Maxillofac Surg. 2008;46:15-21.

11. Lopez-Jornet P, Camacho-Alonso F. Application of a motivation-behaviora skills protocol in gingival lichen planus: a short-term study. J Periodontol. 2010;81:1449-1454.

12. Thorat MS, Raju A, Pradeep AR. Pemphigus vulgaris: effects on periodontal health. J Oral Sci. 2010;52:449-454.

13. Lim HD, Kang JK, Lee YM, Shim YJ. The correlation between desquamative gingivitis associated diseases and plaque-induced periodontal disease. $J$ Oral Med Pain. 2015;40:135-139.

14. Salgado DS, Jeremias F, Capela MV, Onofre MA, Massucato EM, Orrico SR. Plaque control improves the painful symptoms of oral lichen planus gingival lesions. A short-term study. J Oral Pathol Med. 2013;42:728-732.

15. Ertugrul AS, Dursun R, Dundar N, Avunduk MC, Hakki SS. MMP-1, MMP9 and TIMP-1 levels in oral lichen planus patients with gingivitis or periodontitis. Arch Oral Biol. 2013;58:843-852. 